\title{
Effective distributed convolutional neural network architecture for remote sensing images target classification with a pre-training approach
}

\author{
LI Binquan ${ }^{1, *}$ and $\mathrm{HU}$ Xiaohui $^{2}$ \\ 1. School of Automation Science and Electrical Engineering, Beihang University, Beijing 100191, China; \\ 2. Institute of Software, Chinese Academy of Sciences, Beijing 100190, China
}

\begin{abstract}
How to recognize targets with similar appearances from remote sensing images (RSIs) effectively and efficiently has become a big challenge. Recently, convolutional neural network (CNN) is preferred in the target classification due to the powerful feature representation ability and better performance. However, the training and testing of CNN mainly rely on single machine. Single machine has its natural limitation and bottleneck in processing RSIs due to limited hardware resources and huge time consuming. Besides, overfitting is a challenge for the CNN model due to the unbalance between RSIs data and the model structure. When a model is complex or the training data is relatively small, overfitting occurs and leads to a poor predictive performance. To address these problems, a distributed CNN architecture for RSIs target classification is proposed, which dramatically increases the training speed of CNN and system scalability. It improves the storage ability and processing efficiency of RSIs. Furthermore, Bayesian regularization approach is utilized in order to initialize the weights of the CNN extractor, which increases the robustness and flexibility of the CNN model. It helps prevent the overfitting and avoid the local optima caused by limited RSI training images or the inappropriate CNN structure. In addition, considering the efficiency of the Naïve Bayes classifier, a distributed Naïve Bayes classifier is designed to reduce the training cost. Compared with other algorithms, the proposed system and method perform the best and increase the recognition accuracy. The results show that the distributed system framework and the proposed algorithms are suitable for RSIs target classification tasks.
\end{abstract}

Keywords: convolutional neural network (CNN), distributed architecture, remote sensing images (RSIs), target classification, pre-training.

DOI: $10.21629 / J S E E .2019 .02 .02$

\section{Introduction}

With the rapid progress in the remote sensing technology

\footnotetext{
Manuscript received September 11, 2017.

*Corresponding author.

This work was supported by the National Natural Science Foundation of China (U1435220).
}

and the reduction of acquisition costs, a large number of remote sensing images (RSIs) of the Earth are available each day. They are taken from satellites, airplanes or unmanned aerial vehicles (UAVs), with flexible and varied modalities, and spatial and spectral resolutions.

In recent years, deep learning methods $[1,2]$, such as convolutional neural network $(\mathrm{CNN})$, are preferred to the RSIs classification tasks. All these studies reveal that the feature representation of a deep architecture achieves better performance than traditional approaches [3-10]. Compared with hand-crafted features, such as histogram of oriented gradient (HOG) and scale-invariant feature transform (SIFT), CNN is able to automatically learn multiple stages of invariant features for the specific task and has enjoyed the success in a great deal of applications. CNN shows a strong robustness against geometric distortions, such as shifts, scaling and inclination, due to the hierarchical learning structure. Furthermore, unlike many traditional methods, CNN can learn features automatically for RSIs classification, which is suitable for real-time applications.

However, traditional deep learning algorithms by the single machine applied to RSIs show inadequacies in computational capability and storage ability. The storage and processing of RSIs are time-consuming with personal computer due to the limitations of both hardware and software resources. CNN can also be trained with the graphics processing unit (GPU); however, the major drawback of the GPU is similar to the single machine that is hopeless to handle huge computation and huge storage/memory cost. Therefore, clusters of computers and distributed systems are no doubt the promising choices $[11-14]$. The key to implementing distributed processing of RSIs is developing architecture in a parallel manner, as well as providing an effective distributed storage platform for RSIs data [15,16].

As a scalable framework, the distributed architecture has been widely utilized for various tasks. Chu et al. 
[17] demonstrated that the MapReduce framework is suitable for some machine learning techniques including support vector machine (SVM), logistics regression (LR) and Naïve Bayes.

Motivated by the powerful data processing ability of the distributed framework and the excellent performance of CNN, a distributed framework is proposed for RSIs target classification tasks.

Nevertheless, the training of CNN encounters some limitations. For example, the training of fully connected layers may suffer from overfitting problems and local minima. Especially, the CNN model must control its parameters properly when trained from small data sets. As a result, with the limited RSI training images or an unsuitable CNN structure, the balance between model complexity and deep architecture should be properly controlled.

To address this problem, Bayesian regularization is combined to pre-train the networks and initialize the weights. Bayesian approach can potentially avoid the above pitfalls in training neural networks $[18,19]$. Bayesian principle can not only automatically infer hyperparameters by marginalizing them out of the posterior distribution, but also naturally account for the uncertainty in parameter estimates and propagate the uncertainty to predictions. Furthermore, Bayesian techniques are often more robust to overfitting since they average over values of parameters rather than choose a single point estimate.

For the classifier designing, a distributed Naïve Bayes classifier is proposed in order to increase the training speed.

Above all, our contributions can be summarized as follows. Firstly, a distributed CNN architecture is proposed for training the networks, which dramatically increases the training speed and system scalability. It improves the storage ability and processing efficiency of RSIs. Secondly, a pre-training algorithm is proposed to increase the robustness, flexibility and classification precision of CNN. It prevents the training from overfitting and local minima. Thirdly, a distributed Naïve Bayes algorithm using MapReduce is proposed in order to decrease the training cost and increase the efficiency of the classifier. Compared with other algorithms, the distributed system and the proposed method are suitable for RSIs target classification tasks.

The remainder of this paper is constructed as follows. In Section 2, the distributed RSIs target classification systems are proposed. The experiment and analysis are presented in Section 3. Finally, this paper is concluded in Section 4.

\section{Framework of distributed RSIs target classification system}

The distributed RSIs target classification system is illustrated in Fig. 1. It consists of two stages: training and recognition. In the training stage, training samples are trained from the feature extractor to the classifier. In the recognition stage, images are sent to the trained framework for recognition.

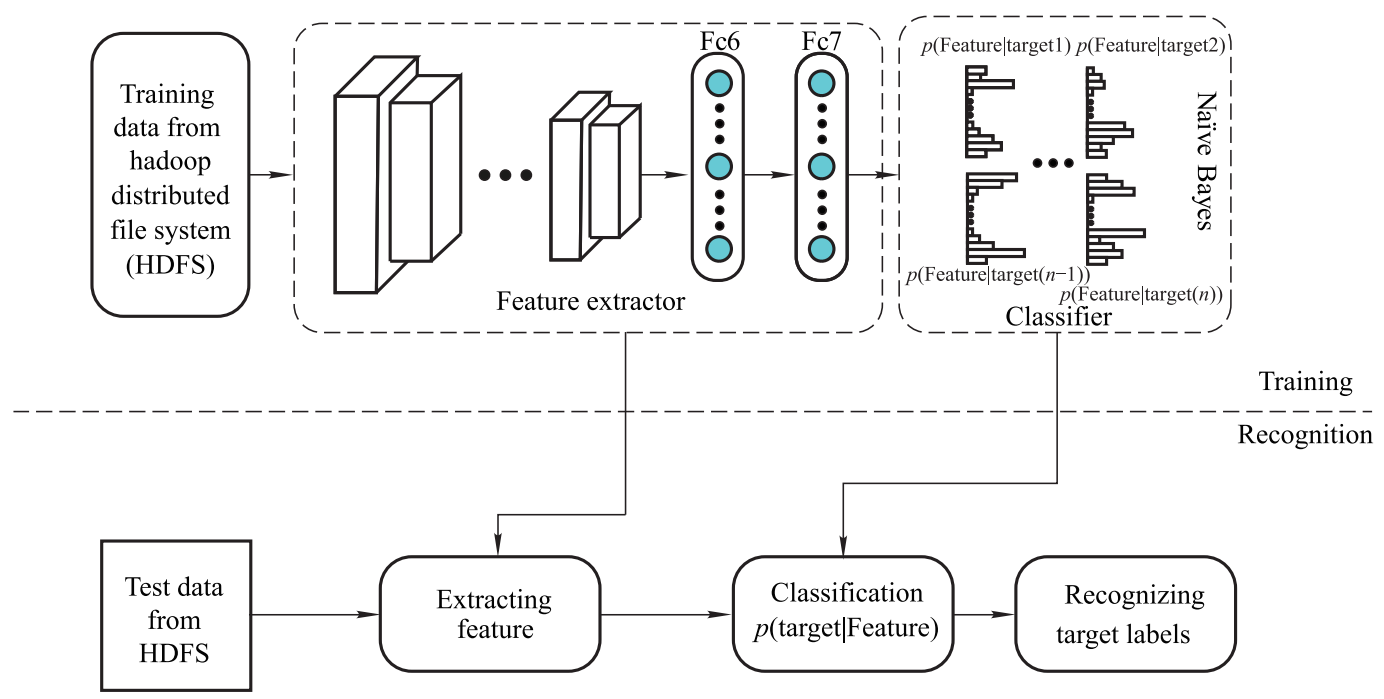

Fig. 1 Overview of distributed RSIs target classification framework

\subsection{Implementation of pre-training strategy}

The training stage consists of the feature extractor and the Naïve Bayes classifier. The feature extractor can extract local features and global features. We utilize convolutional layers to obtain local features. Global features are extracted by fully connected layers. Then the CNN feature vectors of RSIs are sent to the Naïve Bayes classifier. Although backpropagation is widely used in the training of neural networks [20], it still has some disadvantages, such as overfitting the training data. To address the problems, the Bayesian approach is introduced as pre-training 
to learn fully connected layers.

The objective function $F$ in the training procedure of RSIs [21] can be given by

$$
\begin{gathered}
F=\beta E_{D}+\alpha E_{W}= \\
\beta \cdot \frac{1}{n} \sum_{i=1}^{n}(t(i)-a(i))^{2}+\alpha \cdot \frac{1}{m} \sum_{j=1}^{m} w(j)^{2}
\end{gathered}
$$

where $E_{W}$ represents the sum of squared weights of the network, $E_{D}$ represents the training errors of the RSIs, $\alpha$ and $\beta$ are super parameters. Besides, $n$ is the number of training set of RSIs, $m$ is the number of weights, $t(i)$ is the label of RSI, $w(j)$ denotes the weights to be trained and $a(i)$ represents the output of the CNN model.

Then the posterior distribution of weights can be constructed as follows:

$$
p(W \mid D, \alpha, \beta, H)=\frac{p(D \mid W, \beta, H) p(W \mid \alpha, H)}{p(D \mid \alpha, \beta, H)}
$$

where $H$ is the model of $\mathrm{CNN}$, and $p(W \mid \alpha, H)$ represents the prior distribution of weights. $p(D \mid W, \beta, H)$ is the likelihood function given the training samples of RSIs. $p(D \mid \alpha, \beta, H)$ is a normalization factor.

Moreover, the posterior distribution of $\alpha$ and $\beta$ can also be expressed as follows:

$$
p(\alpha, \beta \mid D, H)=\frac{p(D \mid \alpha, \beta, H) p(\alpha, \beta \mid H)}{p(D \mid H)} .
$$

Obviously, the maximum a posteriori (MAP) of (3) can be computed by maximizing $p(D \mid \alpha, \beta, H)$ that is the normalization factor of (2).

According to [22], given the training samples of RSIs and the CNN model, the optimal values for $\alpha$ and $\beta$ can be calculated as follows:

$$
\begin{aligned}
\alpha_{M P} & =\frac{\gamma}{2 E_{W}\left(W_{M P}\right)} \\
\beta_{M P} & =\frac{n-\gamma}{2 E_{D}\left(W_{M P}\right)}
\end{aligned}
$$

where $\gamma=m-2 \alpha_{M P} \operatorname{tr}\left(\nabla^{2} \boldsymbol{F}\left(W_{M P}\right)\right)^{-1}$ is the number of effective weights in the CNN model. $\nabla^{2} \boldsymbol{F}\left(W_{M P}\right)$ is the Hessian matrix of the objective function that can be computed by Gauss-Newton approximation.

After the super parameters $\alpha$ and $\beta$ are obtained, the pre-training is completed. Then, the distributed CNN architecture is utilized to train the network.

\subsection{Distributed CNN architecture for RSIs training}

CNN shows powerful ability in many computer vision applications $[23,24]$. However, the training of $\mathrm{CNN}$ is time consuming. Therefore, the MapReduce based CNN (MR$\mathrm{CNN}$ ) is proposed to reduce computational cost and increase training efficiency.

The parameters of MRCNN are shown in Table 1.
Table 1 Parameters of MRCNN

\begin{tabular}{ccc}
\hline \multirow{2}{*}{ Procedure } & \multicolumn{2}{c}{ Data } \\
\cline { 2 - 3 } Map stage & $\begin{array}{c}\text { Input } \\
\text { training sample }\rangle\end{array}$ & Output \\
\hline \multirow{2}{*}{ Reduce stage } & $\langle$ weight $w$, local $\Delta w\rangle$ & $\langle$ weight $w$, global $\Delta w\rangle$ \\
\hline Main & $\begin{array}{c}\langle\text { training sample, } \\
\text { parameters of network }\rangle\end{array}$ & $\langle$ weight file $\rangle$ \\
\hline
\end{tabular}

As shown in Fig. 2, the MRCNN can be implemented as follows.

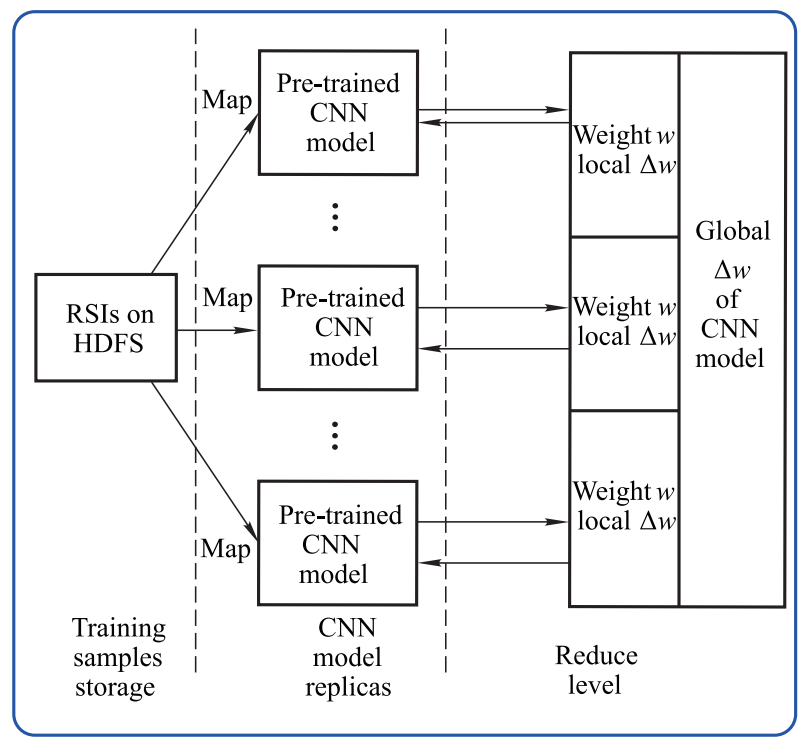

Fig. 2 Architecture of distributed CNN for RSIs training

Algorithm 1 Training RSIs by distributed CNN architecture

Main:

While (the precision of the network is not better than the expected precision)

(i) Run the job by the map stage;

(ii) Execute with the reduce stage;

(iii) Do a batch update on weights of the network for RSIs training and output to the weight file.

End while

Map stage:

(i) Load the parameters of the pre-trained CNN model;

(ii) Read each training sample of RSIs and output the $\langle$ key, value $\rangle$ pairs as $\langle$ target Class ID, training sample $\rangle$;

(iii) Feedforward using value as input;

(iv) Back propagation and output the local $\Delta w$;

(v) Output the $\langle$ key, value $\rangle$ pairs as $\langle$ weight $w$, local $\Delta w\rangle$;

Reduce stage:

(i) Reduce by key and output the global $\Delta w$ of the CNN model; 
(ii) Output the $\langle$ key, value $\rangle$ pairs as $\langle$ weight $w$, global $\Delta w\rangle$.

\subsection{Designing of MapReduce based Naïve Bayes classifier}

In machine learning, Naïve Bayes classifiers are a family of simple and efficient probabilistic classifiers based on Bayes' principle with independence assumptions between the features [25].

The CNN feature extracted from each training sample can be represented as $X=\left\{x_{1}, x_{2}, \ldots, x_{n}\right\}$. Suppose there are $m$ possible classes $\left\{C_{1}, C_{2}, \ldots, C_{m}\right\}$. The probability of a new CNN feature $X$ being in class $C_{i}$ can be computed using Bayes' rule:

$$
P\left(C_{i} \mid X\right)=\frac{P\left(X \mid C_{i}\right) P\left(C_{i}\right)}{P(X)} .
$$

Based on the independence assumptions, $P\left(X \mid C_{i}\right)$ can be computed as follows:

$$
\begin{gathered}
P\left(X \mid C_{i}\right)=P\left(x_{1} \mid C_{i}\right) P\left(x_{2} \mid C_{i}\right) \cdots P\left(x_{n} \mid C_{i}\right)= \\
\prod_{j=1}^{n} P\left(x_{j} \mid C_{i}\right) . \\
P\left(x_{j} \mid C_{i}\right) \text { can be calculated by } \\
P\left(x_{j} \mid C_{i}\right)=\frac{s_{i j}}{s_{i}}
\end{gathered}
$$

where $s_{i j}$ is the frequency of $x_{j}$ in $C_{i}$, and $s_{i}$ represents the number of samples in $C_{i}$. Since $P(X)$ is a constant for the known data set size, the CNN feature $X$ will be classified into class $C_{i}$ when

$$
P\left(C_{i} \mid X\right)>P\left(C_{j} \mid X\right), \quad 1 \leqslant j \leqslant m ; j \neq i .
$$

With these estimations, the calculation is essentially a counting problem. This makes MapReduce a suitable distributed framework for the implementation of Naïve Bayesian classifier (NBC).

$P\left(C_{i}\right)$ can be computed by the frequency of $C_{i}$ in training samples. The estimation of $P\left(x_{j} \mid C_{i}\right)$ is computed by the relative frequency of $x_{j}$ in $C_{i}$. The classification problem is then converted to a counting problem on the training and testing data sets. Consequently, the MapReduce based NBC training algorithm is shown in Fig. 3.

Algorithm 2 The MapReduce based NBC algorithm consists of three stages.

Map stage: Read each training sample, and output the $\langle$ key, value $\rangle$ pairs as $\left\langle C_{i},\left(x_{1}, x_{2}, \ldots, x_{n}\right)\right\rangle$.

Combiner stage: The combiner stage is an optimization stage reducing the $\mathrm{I} / \mathrm{O}$ and data transformation between map and reduce tasks. The combiner operation can also be considered as a local reduce job.
It outputs $\langle$ key, value $\rangle$ pairs as $\left\langle\operatorname{class} C_{i},\left(\operatorname{count}\left(x_{1}\right)\right.\right.$, $\left.\left.\operatorname{count}\left(x_{2}\right), \ldots, \operatorname{count}\left(x_{n}\right)\right)\right\rangle$.

Reduce stage: Reduce by $\operatorname{class} C_{i}$, and compute the global frequency of $x_{j}$ in $C_{i}$. The output $\langle$ key, value $\rangle$ pairs are as follows:

$\left\langle\right.$ class $C_{i}$, (globalfrequency $\left(x_{1}\right)$, globalfrequency $\left(x_{2}\right), \ldots$, globalfrequency $\left.\left.\left(x_{n}\right)\right)\right\rangle$.

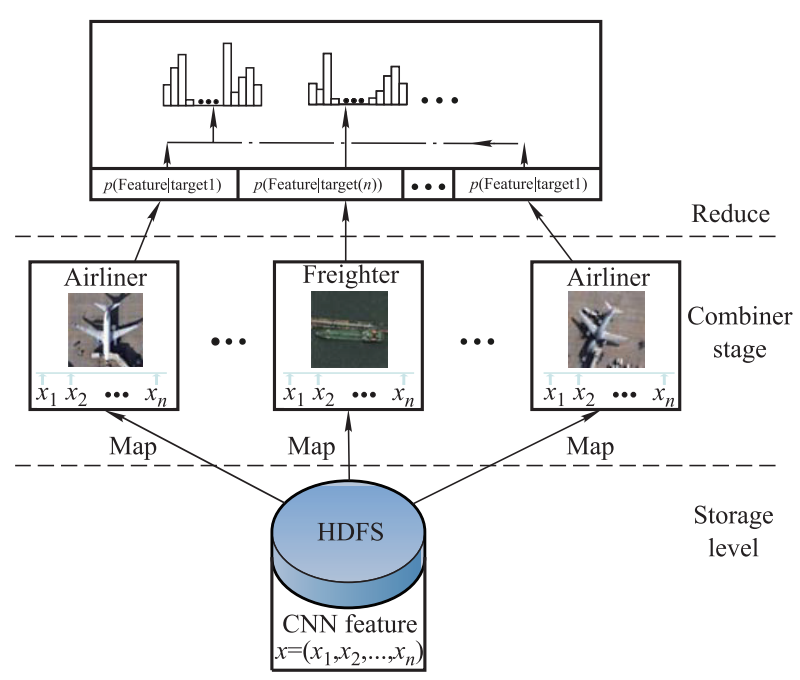

Fig. 3 MapReduce based NBC

\section{Experiment and analysis}

Our experiments are performed on a cluster of machines that have one master and four slaves. The master is configured to use four CPU, 8 GB of RAM. Each salve is configured to use four CPU, $8 \mathrm{~GB}$ of RAM and $750 \mathrm{G}$ disk spaces. All our experimental data are stored in HDFS.

\subsection{Data sets description}

There are 15 classes of training images with $256 \times 256$ pixels in the data sets: Helicopter-1, Helicopter-2, Early warning aircraft, Bomber-1, Bomber-2, Bomber-3, Fighter-1, Fighter-2, Fighter-3, Transport plane-1, Transport plane-2, Airliner, Warship, Aircraft carrier, Freighter. The images contain different resolutions in order to increase the data complexity and diversity. Some samples are shown in Fig. 4, from left to right, up to down: Helicopter-1, Helicopter-2, Early warning aircraft, Bomber-1, Bomber-2, Bomber-3, Fighter-1, Fighter-2, Fighter-3, Transport plane-1, Transport plane-2, Airliner, Warship, Aircraft carrier, Freighter. 

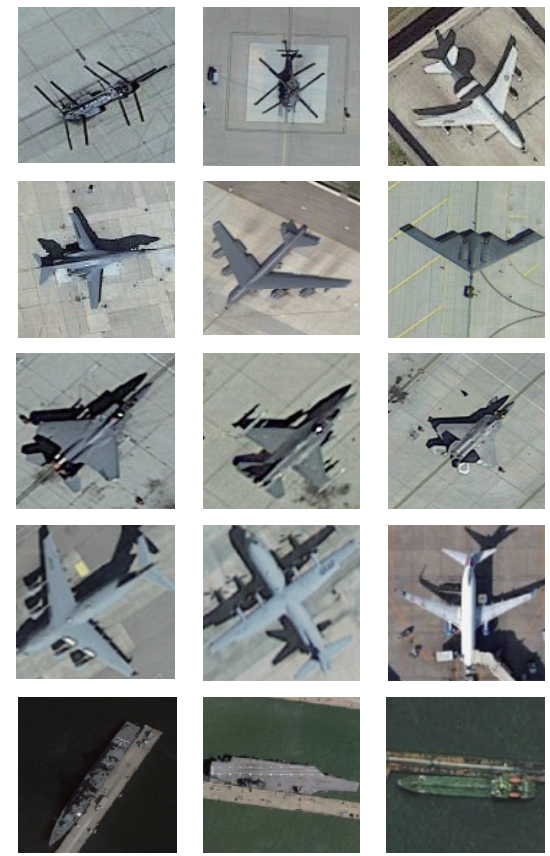

Fig. 4 Some samples of data sets

The training sets contain about $80 \%$ of images in each class. It also increases the capacity of data sets by mirror operation and rotation at $45^{\circ}, 90^{\circ}, 135^{\circ}, 180^{\circ}, 225^{\circ}, 270^{\circ}$, and $315^{\circ}$. Then the data sets are shown in Table 2.

Table 2 Data sets details

\begin{tabular}{ccc}
\hline Target & Training sample & Testing sample \\
\hline Helicopter-1 & 10 & 3 \\
Helicopter-2 & 15 & 4 \\
Early warning aircraft & 20 & 5 \\
Bomber-1 & 29 & 8 \\
Bomber-2 & 21 & 5 \\
Bomber-3 & 4 & 1 \\
Fighter-1 & 12 & 3 \\
Fighter-2 & 19 & 5 \\
Fighter-3 & 5 & 1 \\
Transport plane-1 & 40 & 10 \\
Transport plane- & 36 & 9 \\
Airliner & 284 & 71 \\
Warship & 60 & 15 \\
Aircraft carrier & 8 & 2 \\
Freighter & 214 & 53 \\
Total & $777 \times 8=6216$ & $195 \times 8=1560$ \\
\hline
\end{tabular}

\subsection{Experiment results}

As shown in Table 3, the results illustrate that using the pre-training approach is an effective way to achieve a higher accuracy. The confusion matrix and classification accuracies are reported in Table 4, Table 5 and Fig. 5, from category 1 to 15: Helicopter-1, Helicopter-2, Early warning aircraft, Bomber-1, Bomber-2, Bomber-3, Fighter-1, Fighter-2, Fighter-3, Transport plane-1, Transport plane-2, Airliner, Warship, Aircraft carrier, Freighter. In Fig. 5, category 16 represents the average value.
Table 3 Comparison with different methods

\begin{tabular}{cccc}
\hline \multirow{2}{*}{ Performance } & \multicolumn{4}{c}{ Method } \\
\cline { 2 - 4 } & CNN+Pre-training & CNN & SVM+SIFT \\
\hline $\begin{array}{l}\text { Average value } \\
\text { of accuracy/\% }\end{array}$ & 93 & 86 & 76 \\
\hline
\end{tabular}

Table 4 Confusion matrix of CNN+Pre-training

\begin{tabular}{|c|c|c|c|c|c|c|c|c|c|c|c|c|c|c|c|}
\hline \multirow{2}{*}{ Actual } & \multicolumn{15}{|c|}{ Predicted } \\
\hline & 1 & 2 & 3 & 4 & 5 & 6 & 7 & 8 & 9 & 10 & 11 & 121 & 131 & $14 \quad 1$ & 15 \\
\hline 1 & 96 & 4 & & & & & & & & & & & & & \\
\hline 2 & 9 & 91 & & & & & & & & & & & & & \\
\hline 3 & & & 90 & & & & & & & 3 & & 8 & & & \\
\hline 4 & & & & 91 & & & 2 & 8 & & & & & & & \\
\hline 5 & & & 5 & & 88 & & & & & 8 & & & & & \\
\hline 6 & & & & & & 100 & & & & & & & & & \\
\hline 7 & & & & & & & 88 & & 13 & & & & & & \\
\hline 8 & & & & 3 & & & & 90 & 3 & & & & & & \\
\hline 9 & & & & & & & 13 & & 88 & & & & & & \\
\hline 10 & & & 3 & & 6 & & & & & & 1 & & & & \\
\hline 11 & & & & & & & & & & & 97 & & & & \\
\hline 12 & & & 2 & & 1 & & & & & & & 97 & & & \\
\hline 13 & & & & & & & & & & & & & 96 & 4 & \\
\hline 14 & & & & & & & & & & & & & 6 & 94 & \\
\hline 15 & & & & & & & & & & & & & & & 98 \\
\hline
\end{tabular}

Table 5 Confusion matrix of CNN

\begin{tabular}{|c|c|c|c|c|c|c|c|c|c|c|c|c|c|c|c|}
\hline \multirow{2}{*}{ Actual } & \multicolumn{15}{|c|}{ Predicted } \\
\hline & 1 & 2 & 3 & 4 & 5 & 6 & 7 & 8 & 9 & 10 & 11 & 12 & 13 & 14 & $\overline{15}$ \\
\hline 1 & 83 & 17 & & & & & & & & & & & & & \\
\hline 2 & 16 & 84 & & & & & & & & & & & & & \\
\hline 3 & & & 80 & & 5 & & & & & 3 & & 13 & & & \\
\hline 4 & & & & 86 & & & 8 & 5 & 2 & & & & & & \\
\hline 5 & & & 5 & & 78 & & & & & 13 & & 5 & & & \\
\hline 6 & & & & & & 100 & & & & & & & & & \\
\hline 7 & & & & & & & 79 & 4 & 17 & & & & & & \\
\hline 8 & & & & & & & 13 & 83 & 5 & & & & & & \\
\hline 9 & & & & & & & 25 & & 75 & & & & & & \\
\hline 10 & & & 3 & & 11 & & & & & 86 & & & & & \\
\hline 11 & & & 3 & & & & & & & 4 & 93 & & & & \\
\hline 12 & & & 4 & & 2 & & & & & & & 94 & & & \\
\hline 13 & & & & & & & & & & & & & 91 & 9 & \\
\hline 14 & & & & & & & & & & & & & 197 & 75 & 6 \\
\hline 15 & & & & & & & & & & & & & & 1 & 97 \\
\hline
\end{tabular}

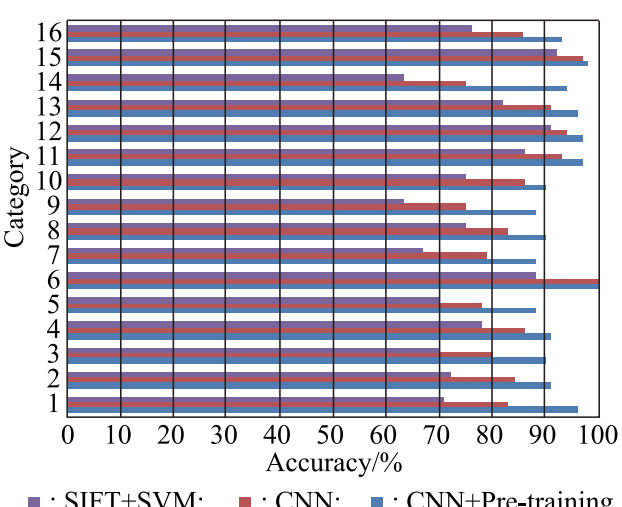

Fig. 5 Accuracy details with different methods 
As shown above, the distinction between aircraft and ship class is relatively high. For the ship class, the confusion between warship and aircraft carrier is higher than that between them and Freighter. For the airplanes class, there is a high degree of discrimination between helicopters and other types of aircraft. However, there is some confusion between Helicopter-1 and Helicopter-2. Early warning aircraft and Airliner have a certain degree of confusion. As the bombers vary in appearance, they will be confused with other aircraft types whose shapes are similar to them, such as Bomber- 1 and the fighters, Bomber- 2 and transportation planes. For the fighter sub-category, because the fighters' appearances are very similar to each other, the confusion between various fighters is obviously higher. In the following, there is a certain degree of confusion between transportation airplanes due to the similar shape. For airliners, there is a certain confusion between them and the Early warning aircraft. In general, $\mathrm{CNN}$ achieves more discriminative feature representations than hand-crafted features. It performs better than the methods without pre-training.

Besides, the computational cost is computed with distributed system or not. As shown in Table 6 and Fig. 6, the distributed system reduces the computational cost in both the training and testing stages. It increases the processing efficiency and is suitable for RSIs target classification tasks.

Table 6 Computational cost of training stage

\begin{tabular}{cc}
\hline Training method & Time/h \\
\hline CNN & 12.9 \\
Distributed CNN & 4.2 \\
\hline
\end{tabular}

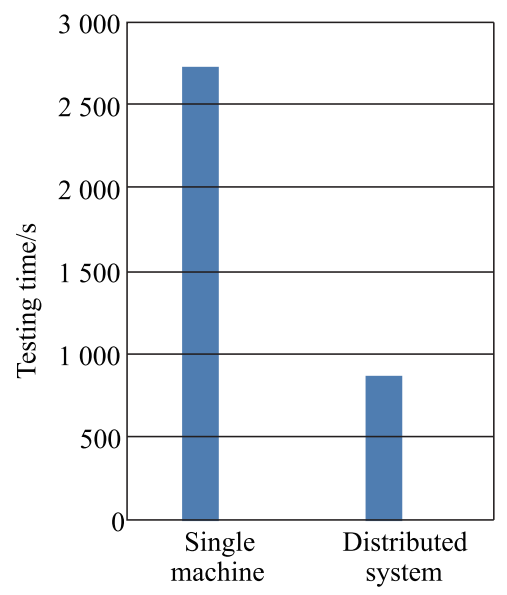

Fig. 6 Processing time of testing stage

\subsection{Robustness evaluation}

To evaluate the robustness of the CNN model, the accuracy by different percentage of training samples is calculated with and without pre-training. As shown in Fig. 7, the pre-training strategy helps CNN achieve better performance, even with only $10 \%$ of the training samples. It reveals that the proposed method is more robust than those without pre-training.

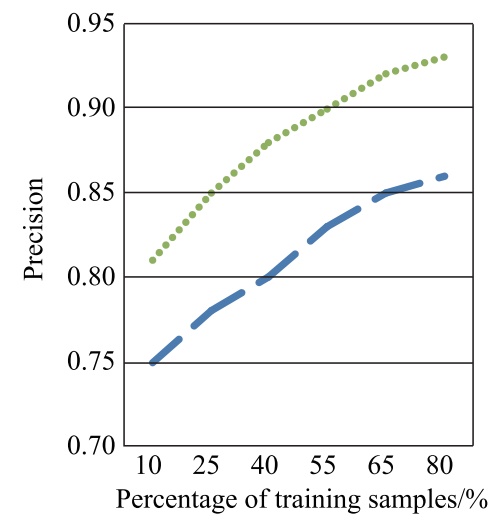

- - : Without pre-training; ……* : With pre-training.

Fig. 7 Robustness evaluation of CNN model in different percentage of training samples

In addition, accuracy with different convolution layers is compared. As shown in Fig. 8, the observation demonstrates that a deeper architecture can provide more discriminative feature representation of RSIs. The CNN model with pre-training method performs robustly due to the automatic adaption of various model structures, even with one convolution layer.

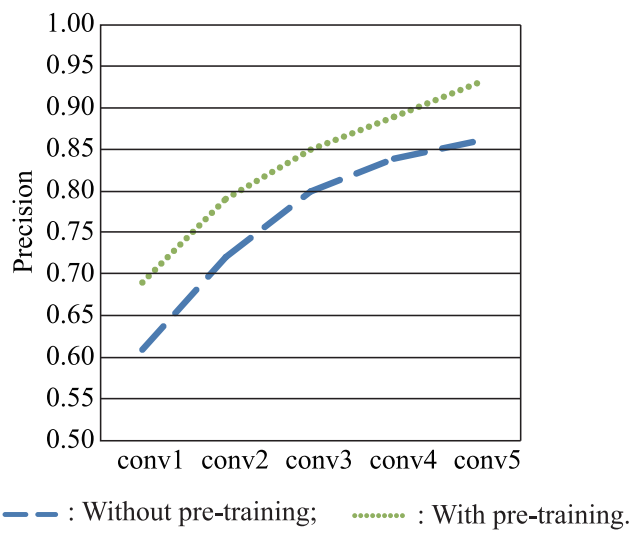

Fig. 8 Robustness evaluation of CNN model with different number of convolution layers

Although there are some existing CNN models that can be fine-tuned, the fine-tuned CNN models are not flexible enough to some complex applications in RSI target classification, such as various types of targets, varied CNN models or limited training data. Our method is more flexible and more robust due to the automatic adaption of various model structures, even with one convolution layer or limited training data. In addition, unlike other methods deploying the single machine, the proposed distributed system reduces 
the computational cost and increases the processing efficiency simultaneously in both training and testing stages. Moreover, considering the computation and system scalability of the distributed architecture, the proposed method and system can be deployed on larger clusters in order to further improve the storage ability and processing efficiency, which is more suitable for real-world RSIs classification tasks.

\section{Conclusions}

A distributed CNN architecture with the pre-training strategy is proposed for RSIs target classification tasks. Compared with other algorithms, the proposed method is more flexible, more robust and increases the recognition accuracy. The distributed system reduces the computational cost in both training and testing stages, improving the storage ability and processing efficiency of RSIs. It is suitable for real-world RSIs target classification tasks.

\section{References}

[1] LECUN Y, KAVUKCUOGLU K, FARABET C. Convolutional networks and applications in vision. Proc. of the IEEE International Symposium on Circuits \& Systems, 2010: 253 256.

[2] SERMANET P, EIGEN D, ZHANG X, et al. OverFeat: integrated recognition, localization and detection using convolutional networks. Proc. of the International Conference on Learning Representations, 2014: 1-16.

[3] ZEILER M D, FERGUS R. Visualizing and understanding convolutional networks. Proc. of the European Conference on Computer Vision, 2014: 818-833.

[4] ZHANG F, DU B, ZHANG L. Saliency-guided unsupervised feature learning for scene classification. IEEE Trans. on Geoscience \& Remote Sensing, 2015, 53(4): 2175-2184.

[5] HU F, XIA G, WANG Z, et al. Unsupervised feature learning via spectral clustering of multidimensional patches for remotely sensed scene classification. IEEE Journal of Selected Topics in Applied Earth Observations \& Remote Sensing, 2015, 8(5): $2015-2030$.

[6] ZHAO L J, TANG P, HUO L Z. Land-use scene classification using a concentric circle-structured multi-scale bag-of-visualwords model. IEEE Journal of Selected Topics in Applied Earth Observations \& Remote Sensing, 2014, 7(12): 46204631.

[7] CHEN S, TIAN Y. Pyramid of spatial relations for scene-level land use classification. IEEE Trans. on Geoscience \& Remote Sensing, 2015, 53(4): $1947-1957$.

[8] PENATTI O A, NOGUEIRA K, SANTOS J A D. Do deep features generalize from everyday objects to remote sensing and aerial scenes domains? Proc. of the IEEE Conference on Computer Vision and Pattern Recognition, 2015: 44-51.

[9] MARMANIS D, DATCU M, ESCH T, et al. Deep learning earth observation classification using image net pre-trained networks. IEEE Geoscience \& Remote Sensing Letters, 2016, 13(1): $105-109$.

[10] SCOTT G J, ENGLAND M R, STARMS W A, et al. Training deep convolutional neural networks for land-cover classification of high-resolution imagery. IEEE Geoscience \& Remote Sensing Letters, 2017, 14(4): 549-553.

[11] BAO H C, FANG L, LIU R Y. Research and application of the storage way of land use change records. Journal of Zhejiang University (Science Edition), 2011, 38(2): 218-222. (in
Chinese)

[12] LAI J B, LUO X L, YU T, et al. Remote sensing data organization model based on cloud computing. Computer Science, 2013, 40(7): 80-84.

[13] YANG H P, SHEN Z F, LUO J C, et al. Recent developments in high performance geocomputation for massive remote sensing data. Journal of Geo-Information Science, 2013, 15(1): 128 136. (in Chinese)

[14] LIU Y, GUO W, JIANG W S, et al. Research of remote sensing service based on cloud computing mode. Application Research of Computers, 2009, 26(9): 3428 - 3431. (in Chinese)

[15] REN F H, WANG J N. Turning remote sensing to cloud services: technical research and experiment. Journal of Remote Sensing, 2012, 16(6): 1339-1346. (in Chinese)

[16] WAN B, YANG L. Data center: GIS function warehouse. Earth Science-Journal of China University of Geosciences, 2010, 35(3): 357 -361. (in Chinese)

[17] CHU C, KIM S K, LIN Y A, et al. Map-reduce for machine learning on multicore. Proc. of Advances in Neural Information Processing Systems, 2007: $281-288$.

[18] MACKAY D J C. A practical Bayesian framework for back propagation networks. Neural Computation, 1992, 4(3): 448472.

[19] SNOEK J, LAROCHELLE H, ADAMS R P. Practical Bayesian optimization of machine learning algorithms. Proc. of Advances in Neural Information Processing Systems, 2012: $2951-2959$.

[20] RUMELHART D, HINTON G, WILLIAMS R. Learning representations by back-propagating errors. Nature, 1986, 323(6088): $533-536$.

[21] XU M, ZENG G, XU X, et al. Application of Bayesian regularized BP neural network model for trend analysis, acidity and chemical composition of precipitation in north Carolina. Water, Air, and Soil Pollution, 2006, 172(1-4): 167-184.

[22] FORESEE F D, HAGAN M T. Gauss-Newton approximation to Bayesian regularization. Proc. of the International Joint Conference on Neural Networks, 1997: 1930-1935.

[23] ERHAN D. Why does unsupervised pre-training help deep learning? Journal of Machine Learning Research, 2010, 11(3): $625-660$.

[24] FAN J, XU W, WU Y, et al. Human tracking using convolutional neural networks. IEEE Trans. on Neural Network, 2010, 21(10): $1610-1623$.

[25] LIU B, BLASCH E, CHEN Y, et al. Scalable sentiment classification for big data analysis using Naïve Bayes classifier. Proc. of the IEEE International Conference on Big Data, 2013: $99-104$.

\section{Biographies}

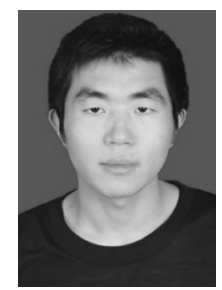

LI Binquan was born in 1986. He is now pursuing his Ph.D. degree with School of Automation Science and Electrical Engineering, Beihang University, Beijing, China. His research interests are deeplearning, computer vision and big data processing.

E-mail: jz05022300@sina.com

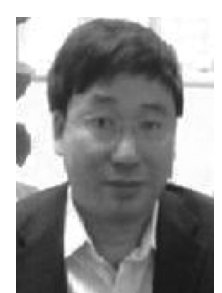

HU Xiaohui was born in 1960. He is a researcher at Institute of Software, Chinese Academy of Sciences. His research interests include artificial intelligence, information system integration and simulation technology.

E-mail: hxh@iscas.ac.cn 